\title{
Evaluación de la oxidación superficial de recubrimientos de Ti6Al4V obtenidos por recubrimiento por láser
}

\author{
V. Amigó*, J.J. Candel*, J.M. Amado** y A. Yañez**
}

\begin{abstract}
Resumen
Se ha estudiado la influencia de los parámetros de procesado sobre la oxidación superficial y propiedades mecánicas de diferentes recubrimientos de Ti6Al4V. La gran reactividad química del polvo de titanio junto a la elevada temperatura que se alcanza producen gran variedad de colores en los óxidos formados. Esta oxidación puede tener un efecto perjudicial sobre el comportamiento en servicio, por lo que su evaluación es fundamental. Aunque la influencia del procesado en el color de los óxidos formados es clara, no existe una relación sencilla que explique sus propiedades mecánicas. Por ese motivo se ha seguido una metodología basada en la microscopia, microanálisis por espectroscopia de longitud de onda (WDS) y nanoindentación. Los resultados indican que no hay diferencias importantes en la dureza y módulo elástico entre dos recubrimientos que presentan diferentes colores de oxidación.
\end{abstract}

\section{Evaluation of superficial oxidation layer of Ti6Al4V laser cladding coatings}

\begin{abstract}
It has been studied the influence of processing parameters on the superficial oxidation and mechanical properties of different coatings of Ti6Al4V. The high chemical reactivity of titanium powder, and the high temperature reached during laser processing, produces a great variety of colors in the oxides formed. This oxidation can have a detrimental effect on the behavior in service so that its evaluation is essential. Although the influence of processing on the color of the oxides formed is clear, there is no simple relationship to explain its mechanical properties. For this reason it has been applied a methodology based on microscopy, microanalysis wavelength spectroscopy (WDS) and nanoindentation. The results indicate no significant differences in hardness and elastic modulus between two coatings with different colors of oxidation.
\end{abstract}

Keywords

Laser cladding; Titanium alloys; Oxidation; Hardness.

\section{INTRODUCCIÓN}

Las aleaciones de titanio se utilizan, ampliamente, en muchas aplicaciones de ingeniería, donde la combinación de propiedades mecánicas y químicas es fundamental. No obstante, bajo condiciones extremas de oxidación, corrosión, desgaste y/o a elevadas temperaturas sufren un rápido deterioro, por lo que desarrollar recubrimientos de elevadas prestaciones, para estas aplicaciones, es fundamental ${ }^{[1]}$.

El laser cladding es una buena alternativa para obtener recubrimientos precisos, libres de defectos y con

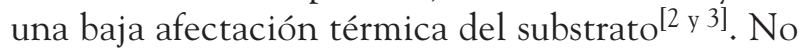
obstante, el titanio presenta una elevada reactividad química con la atmósfera, a elevadas temperaturas
( $\mathrm{T}>600^{\circ} \mathrm{C}$ ), por lo que su procesado por láser puede ser crítico ${ }^{[4]}$.

La reacción del titanio con el oxígeno influye en su resistencia mecánica y ductilidad ${ }^{[5]}$. Por tanto, debe evitarse la oxidación del recubrimiento siempre que el material deba trabajar bajo fatiga.

Los óxidos de titanio presentan una gran variedad de colores y aunque están relacionados con la temperatura y tiempo de oxidación no hay una relación sencilla que permita definir, claramente, si el recubrimiento es correcto o no. Sin embargo, existen normas para soldadura de titanio como la AWS D $17.1^{[6]}$ que proporcionan indicaciones generales para aceptar o no una soldadura por arco eléctrico en función del color obtenido.

\footnotetext{
* ITM-UPV, c/ Vera, s/n, 46022 Valencia., España. e-mail: vamigo@mcm.upv.es.

** Departamento de Ingeniería Industrial II, Universidade da Coruña, Mendizábal s/n, Ferrol E-15403, España. e-mail: jose.amado.paz@udc.es.
} 
En el caso del procesado por láser, el tiempo de interacción es extremadamente pequeño y el calor está muy localizado. Por tanto, las indicaciones generales de soldadura no son aplicables, directamente. Es necesario valorar si las capas coloreadas que aparecen en algunos recubrimientos son críticas para las propiedades mecánicas o si se trata de una leve oxidación superficial que sufre el cordón, una vez ha pasado ya el cabezal y no protege ese punto.

En este trabajo se han obtenido cortes transversales de cordones que presentan diversas coloraciones debidas a la oxidación. Mediante un análisis sistemático con ayuda de microscopia electrónica de barrido, microanálisis químico y medidas de nanoindentación se pretende evaluar si la oxidación es solo superficial o si tiene mayor influencia en la microestructura y propiedades del cordón.

\section{TRABAJO EXPERIMENTAL}

\subsection{Equipo láser empleado y recubrimientos obtenidos}

Para la fabricación de los recubrimientos se utilizó una fuente láser de Nd:YAG de $2.2 \mathrm{~kW}$ en modo continuo, equipada con un cabezal que permite el procesado por laser cladding coaxial mediante soplado de polvo. El gas de protección fue argón 5.0 con un caudal de $10 \mathrm{l} / \mathrm{min}$ y el diámetro de haz (D) de $3 \mathrm{~mm}$.

Se ha diseñado una plantilla de cordones en la que se varía, de forma controlada, la potencia $(P)$ y la velocidad (V) para analizar el efecto de la energía $(\mathrm{E}=\mathrm{P} / \mathrm{VD})$ sobre la oxidación del cordón.

En concreto la ventana de parámetros fue:

- Potencia, entre 1.000 y $2.000 \mathrm{~W}$.

— Velocidad, entre 5 y $10 \mathrm{~mm} / \mathrm{s}$.

\subsection{Definición de materiales}

Se ha utilizado polvo de Ti6Al4V atomizado, de morfología esférica y con una granulometría adecuada para el procesado por láser $(40 \mu \mathrm{m}<\mathrm{d}<75 \mu \mathrm{m})$.

El substrato ha sido una chapa de Ti6Al4V de 3 $\mathrm{mm}$ de espesor y unas dimensiones de 100x150 mm.

En la tabla I se muestra la composición de las materias primas utilizadas.

\subsection{Metodología de caracterización}

Se ha obtenido una imagen general de la plantilla para mostrar la coloración y aspecto de los cordones (Fig. 1).
Tabla I. Composición química (\% en peso) de los materiales empleados

Table I. Chemical composition (\%wt) of raw materials

\begin{tabular}{cccr}
\hline Elemento & Polvo & Substrato & Patrón \\
\hline $\mathrm{Ti}$ & bal & bal & 89,54 \\
$\mathrm{Al}$ & 6,05 & 6,21 & 6,16 \\
$\mathrm{~V}$ & 4,12 & 4,10 & 3,97 \\
$\mathrm{Fe}$ & 0,06 & 0,12 & 0,12 \\
$\mathrm{O}$ & 0,10 & 0,08 & 0,11 \\
\hline
\end{tabular}

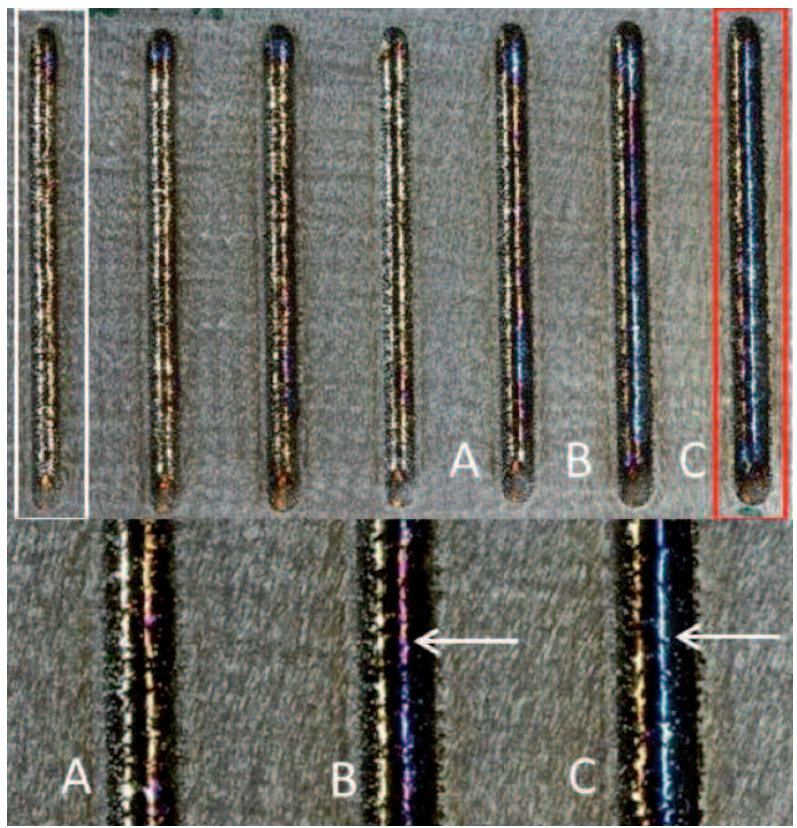

Figura 1. Aspecto general de la plantilla obtenida. Se ha remarcado los cordones 1 y 11 elegidos para el estudio y se ha ampliado las condiciones que presentan coloraciones diferentes debido a los óxidos.

Figure 1. General features of experimental set up. It has been underlined the selected conditions 1 and 11 and it has been enlarged the conditions that present different coloration due to the oxidation.

A continuación, se han preparado muestras metalográficas de los cortes transversales mediante pulido mecánico, llegándose hasta un pulido final con una solución de sílice coloidal, de 0,05 $\mu \mathrm{m}$ (Fig. 2).

Antes de atacar químicamente la superficie, se realizaron medidas de dureza y módulo elástico 


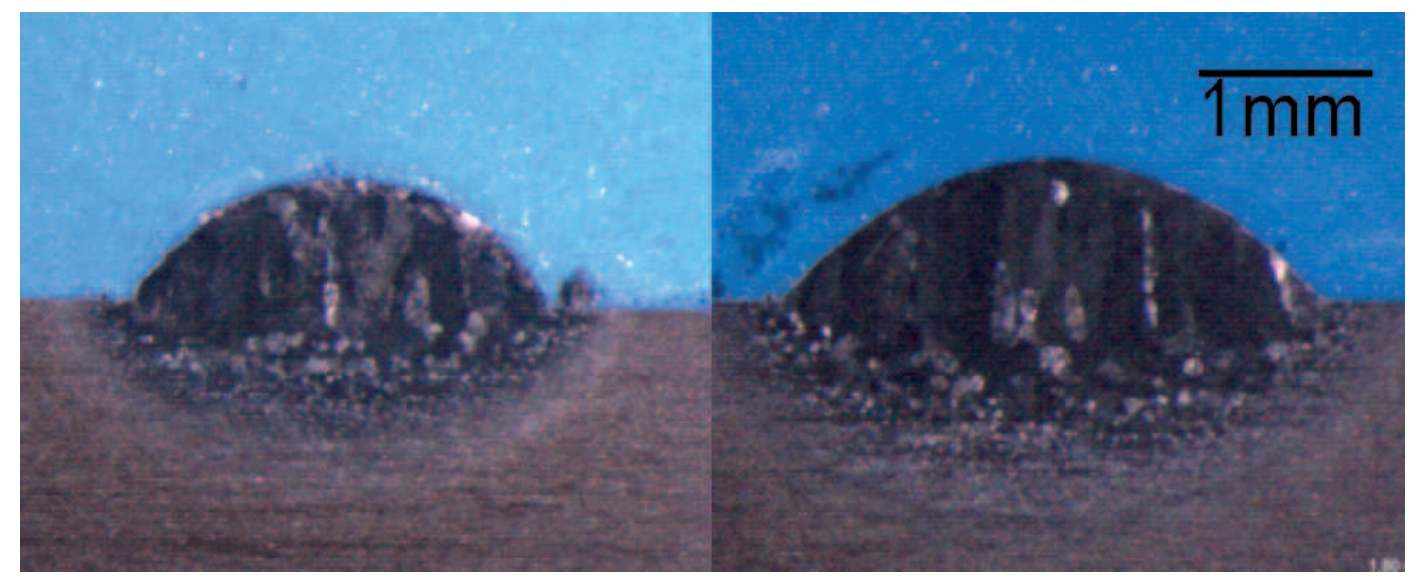

Figura 2. Corte transversal de los cordones 1 y 11 que se han seleccionado.

Figure 2. Cross section of the single tracks 1 and 11 that have been selected.

mediante nanoindentación. En concreto, se ha utilizado una punta Bercovich para obtener huellas de $2.000 \mathrm{~nm}$ de profundidad, agrupadas en matrices de $4 \times 4$, separadas $25 \mu \mathrm{m}$ entre sí y a una distancia de $25 \mu \mathrm{m}$ del punto más alto del cordón (Fig. 3). Para

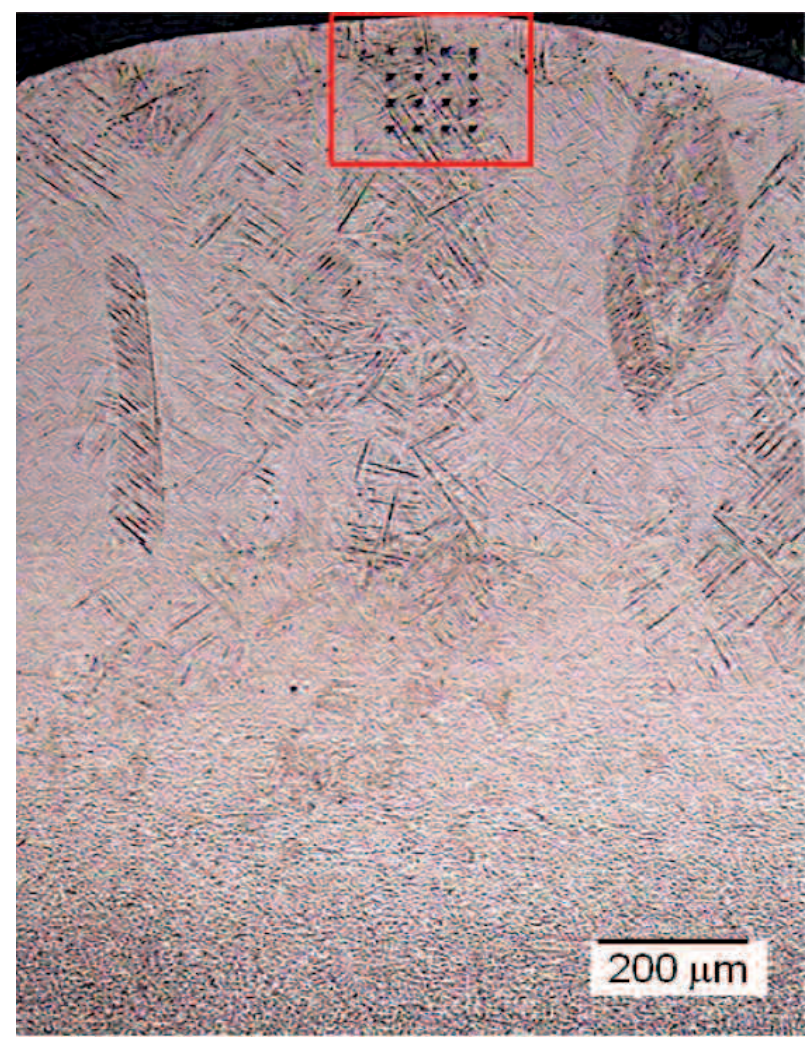

Figura 3. Microestructura del cordon 1 y posicion de la matriz de nanoindentación.

Figure 3. Microstrostructure of single track 1 and location of nanoindentation matrix. el cálculo de dureza y módulo elástico se ha seguido el método de Oliver y Pharr.

A continuación, se observaron las huellas con microscopia electrónica de barrido (SEM) y se analizó la composición del material en la zona adyacente de cada fila de indentaciones (Fig. 4). Los elementos principales ( $\mathrm{Ti}, \mathrm{Al}$ y $\mathrm{V}$ ) se obtuvieron mediante microanálisis de energías dispersivas (EDS) y la contaminación en oxígeno mediante microanálisis

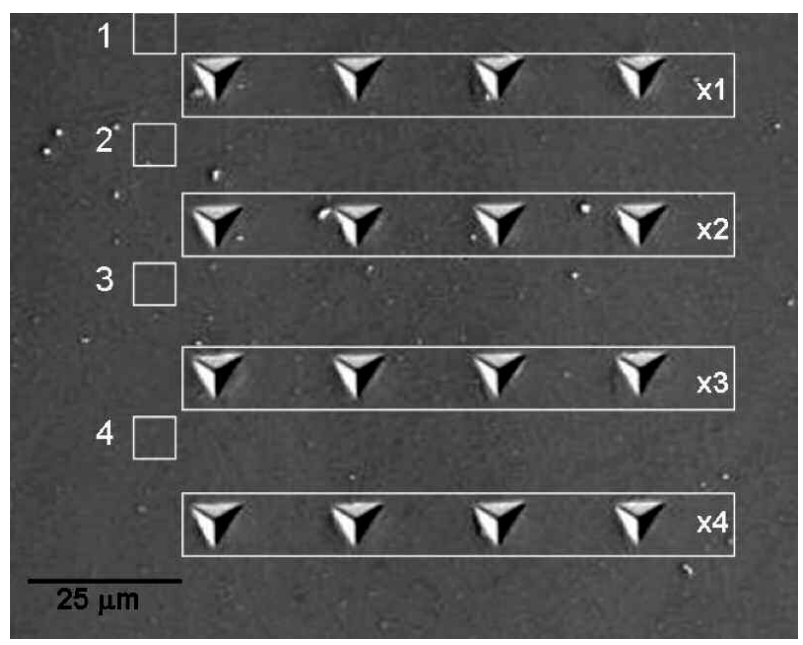

Figura 4. Detalle de la matriz de huellas de nanoindetación. Se ha calculado el promedio de cada fila de huellas $(x 1, x 2, x 3, x 4)$. Se indica mediante un número $(1,2,3,4)$ la posición donde se obtuvieron los espectros de EDS+WDS.

Figure 4. Nanoindentation matrix detail. Average of every file imprints has been calculated ( $x 1$, $x 2, x 3, x 4)$. EDS+EDS spectrum position is indicated by number $(1,2,3,4)$. 
por longitud de onda (WDS), para aumentar la precisión de la cuantificación. Se utilizó, como patrón, una muestra de la zona central de una barra calibrada de Ti6Al4V ELI de composición conocida (Tabla I).

Una vez atacadas las muestras con reactivo Kroll, se realizaron barridos de microdureza Vickers con carga de $200 \mathrm{~g}$ para obtener valores de dureza a escala microscópica y se observó la microestructura mediante microscopía óptica.

\section{RESULTADOS Y DISCUSIÓN}

\subsection{Forma y microestructura de los cordones}

Tras el procesado por láser, se observa que al aumentar la energía aportada, los cordones muestran diversos colores debidos a la formación de óxidos (Fig. 1). De acuerdo con la norma AWS D17.1, los colores violeta y azul son los más perjudiciales puesto que implican una oxidación a mayor temperatura. Los colores observados concuerdan con este criterio ya que el azul coincide con las condiciones en que se aportó más energía.

Para evaluar el efecto de la oxidación, se eligieron dos muestras: la primera, con una energía aportada de 3,4 kJ/ $\mathrm{cm}^{2}$ (M1, P=1.080W; V=10 mm/s), que presentaba color plata; en la segunda, se aportó una energía mayor, de $6,5 \mathrm{~kJ} / \mathrm{cm}^{2}(\mathrm{M} 11, \mathrm{P}=1.560 \mathrm{~W}$; $\mathrm{V}=8 \mathrm{~mm} / \mathrm{s}$ ) y su color era azul oscuro. Ambos cordones tienen una forma adecuada, libre de poros y grietas y están fuertemente unidos al substrato (Fig. 2).

No obstante, las dimensiones del cordón M11 y su zona afectada por el calor (ZAC) son mucho mayores que en el cordón M1 (Tabla II). Esto favorece que el recubrimiento se oxide cuando el cabezal se desplaza a lo largo del cordón, ya que debido a su mayor masa

Tabla II. Dimensiones de los cordones

Table II. Geometrical features of the selected single tracks

\begin{tabular}{lcc}
\hline \multicolumn{1}{r}{ Elemento } & Polvo & Substrato \\
\hline $\mathrm{A}_{\text {clad }}\left(\mathrm{mm}^{2}\right)$ & 1,285 & 1,867 \\
$\mathrm{~A}_{\text {zac }}\left(\mathrm{mm}^{2}\right)$ & 2,042 & 4,133 \\
$\mathrm{H}_{\mathrm{c}}(\mathrm{mm})$ & 0,768 & 0,837 \\
$\mathrm{~W}_{\mathrm{c}}(\mathrm{mm})$ & 0,831 & 1,302 \\
$\mathrm{H}_{\text {zac }}(\mathrm{mm})$ & 2,479 & 3,272 \\
$\mathrm{~W}_{\text {zac }}(\mathrm{mm})$ & 3,184 & 4,253 \\
\hline
\end{tabular}

caliente tendrá una velocidad de enfriamiento mucho menor y un tiempo de permanencia, a temperaturas superiores a $500^{\circ} \mathrm{C}$, mayor.

La microestructura de los cordones (Fig. 3), está formada por agujas de martensita, agrupada en placas dentro de cada grano de titanio beta previo, que se formó. Además, se produce una transición gradual entre el cordón y el substrato sin la aparición de una interfase clara.

\subsection{Análisis de la dureza}

Las imágenes de electrones secundarios de las huellas de nanoindentación muestran que la preparación superficial ha sido correcta, sin rayas o deformación superficial aparente. Se comprueba que algunas huellas presentan acumulación de material alrededor de la huella (fenómeno pop in) lo que indica que el material tiene un comportamiento dúctil ${ }^{[7]}$ (Fig. 4).

Se ha calculado el promedio de las medidas de dureza y módulo elástico para cada fila de huellas (Xi) y los resultados se muestran en la figura 5 . Se observa que para la muestra 1 (E, baja), hay una disminución progresiva de la dureza desde la zona más cercana a la corteza del recubrimiento (x1) hasta la zona más interna $(\mathrm{x} 4)$, mientras que, en la muestra 11, la dureza es prácticamente constante. Este efecto podría estar relacionado con el contenido en oxígeno retenido y/o la mayor velocidad de enfriamiento del cordón M1.

Los valores de microdureza Vickers que se han realizado muestran que en la zona superior del cordón 1 hay un ligero endurecimiento (Tabla III).

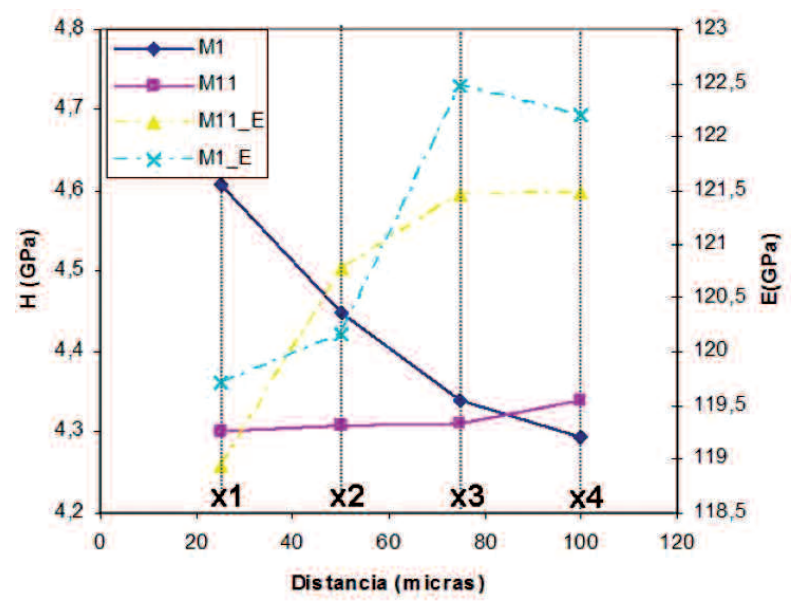

Figura 5. Resultado de la microdureza y módulo elástico obtenido mediante nanoindentación.

Figure 5. Nanohardness and Elastic modulus measurements. 
Tabla III. Valores de microdureza Vickers obtenidos

Table III. Vickers microhardness measurements

\begin{tabular}{ccc}
\hline Elemento & Promedio & Zona Sup. \\
\hline M1 & $418 \pm 18$ & 440 \\
M11 & $412 \pm 19$ & 412 \\
\hline
\end{tabular}

Aunque, en valor promedio, la diferencia entre ambos cordones es mínima. Por tanto, solo existen diferencias apreciables en la capa más superficial. Para explicar este comportamiento es necesario recurrir al análisis mediante EDS+WDS.

\subsection{Microanálisis mediante EDS+WDS}

Los espectros obtenidos muestran que la diferencia en el contenido de elementos de aleación ( $\mathrm{Al}$ y V), entre ambas muestras, es mínima. Tan solo, en la zona 1 (a $15 \mu \mathrm{m}$ de la superficie) existe un pequeño enriquecimiento en aluminio para la muestra 11 , en la que se aportó mucha energía (Fig. 6).

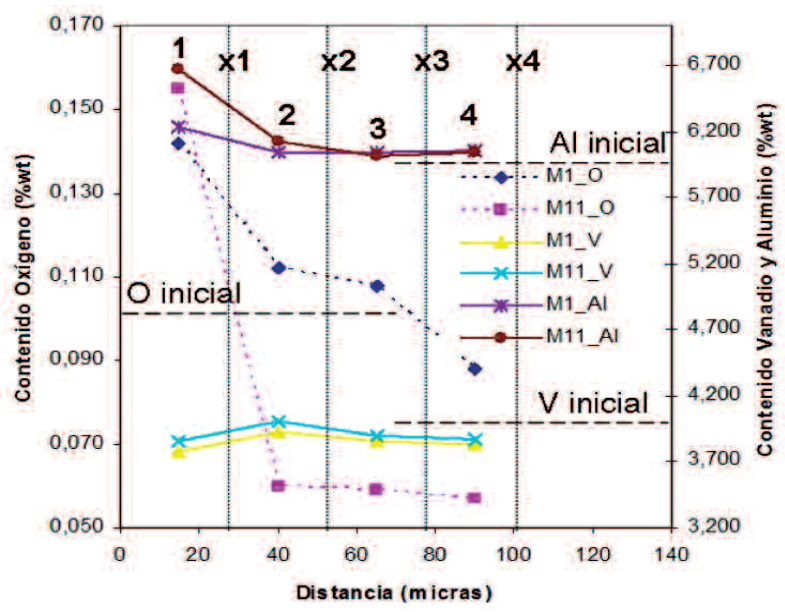

Figura 6. Contenido en oxígeno y otros elementos de aleación de las muestras 1 y 11 . Se han analizado las zonas marcadas en la figura 4. Adicionalmente, se indica con línea punteada los contenidos iniciales del polvo empleado.

Figure 6. Oxygen and other alloying elements content evolution on sample 1 and 11. Analyzed areas were indicated in figure 4. Additional to this is shown with lines the initial content of the
Sin embargo, sí se aprecia diferencia en el contenido en oxígeno. Aunque la muestra 11 presenta mayor cantidad de oxígeno cerca de la superficie (zona 1), el contenido cae bruscamente. En la muestra 1, la evolución es, prácticamente, lineal y el contenido es mayor que en la muestra 11. Este efecto, que aparece en el cordón con alta energía, podría estar relacionado con la disolución del oxígeno en el titanio líquido y su posterior acumulación en la superficie. En este proceso, la velocidad de enfriamiento es el parámetro crítico ya que, si es excesivamente alta, el gas queda atrapado en todo el cordón.

En cualquier caso, las diferencias halladas en dureza de la zona x1 y x2 de ambos cordones no puede ser explicada, sólo, por las diferencias composicionales. Al observar imágenes con mayor magnificación, de estas zonas (Fig. 7), se aprecia, en el cordón 1, unos precipitados oscuros en forma de esferoides y que se acumulan, principalmente, en la parte

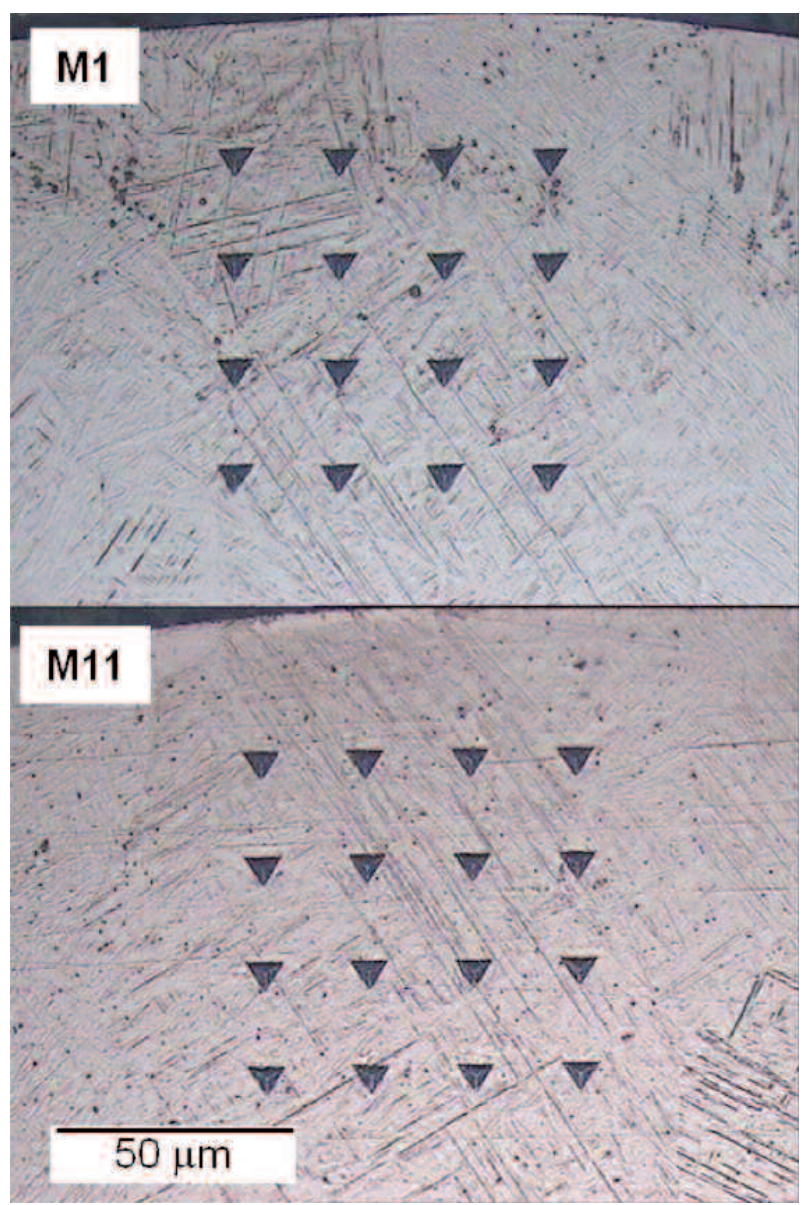

Figura 7. Detalle de los compuestos esféricos encontrados en la parte superior del recubrimiento 1 y 11.

Figure 7. Spherical compounds found in upper area of 1 and 11 single tracks. 
superior. También, están presentes en el cordón 11, pero son de menor tamaño y están más homogéneamente distribuidos.

La formación de precipitados globulares durante los procesos de soldeo suele estar asociada a la contaminación. Pero, no solo es debida a la reactividad con la atmósfera sino que, también, influyen elementos traza como el hierro, el hidrógeno o el carbono ${ }^{[8]}$. La acumulación de estos compuestos en la zona superior del cordón 1 puede ser la causante del ligero endurecimiento (entorno al $10 \%$ del valor promedio) que se ha observado en esta zona.

Por tanto, sólo se han observado diferencias significativas en la capa superior del cordón (de unos 25 $\mu \mathrm{m}$ de espesor), por lo que se trata de un fenómeno superficial que podría eliminarse con un cepillado o rectificado. No obstante, se han estudiado cordones aislados pero, durante el procesado de recubrimientos por solape de cordones, este efecto puede ser mucho más crítico y debería estudiarse en futuros trabajos.

\section{CONCLUSIONES}

Mediante un estudio sistemático de diversos cordones se ha comprobado que el procesado por láser, de las aleaciones de titanio, muestra una complejidad importante debido a la gran reactividad del titanio con la atmósfera.

La utilización conjunta de técnicas de microscopía, microanálisis y nanoindentación ha permitido estudiar el efecto que tiene la oxidación sobre las propiedades del cordón. Esto, demuestra la enorme potencia del método empleado ya que ha permitido detectar elementos traza como el oxígeno.

Contrariamente a lo referido por algunas normas de soldadura por arco eléctrico, la observación del color no basta como criterio para rechazar un cordón obtenido por soldadura láser. El aporte localizado de calor implica grandes velocidades de enfriamiento por lo que, si el proceso está correctamente protegido por el cabezal, sólo puede oxidarse una fina capa, durante pocos segundos.
No se han encontrado diferencias destacables, ni en dureza ni en módulo elástico, entre dos recubrimientos con energía aportada muy diferente. Las pequeñas discrepancias, a nivel sub-superficial, parecen estar más relacionadas con la velocidad de enfriamiento que con la formación de óxidos.

Los resultados sugieren que el titanio es capaz de absorber y diluir con facilidad sus propios óxidos por lo que no se produce un efecto tan perjudicial como el esperado inicialmente.

\section{Agradecimientos}

Los autores agradecen al Ministerio de Ciencia e Innovación por la financiación de esta investigación a través de los proyectos MAT2008-06882-C04-03/04 enmarcados en el Programa de Materiales del Plan Nacional.

\section{REFERENCIAS}

[1] Y.S. Tian, C.Z. Chen, S.T. Li y Q.H. Huo, Appl. Surf. Sci. 242 (2005) 177-184.

[2] S. Yang, W. Liu, M. Zhong y Z. Wang, Mater. Lett. 58 (2004) 2.958-2.962.

[3] I. Vicario, C. Soriano, C. Sanz y R. Bayón, Rev. Metal. Madrid 45 (2009) 14-19.

[4] H. Guleryuz y H. Cimenoglu, Surf. Coat. Technol. 192 (2005) 164-170.

[5] J. Alcisto, A. Enriquez, H. Garcia, S. Hinkson y M. Hahn , Eng. Fail. Analysis 11 (2004) 811 816

[6] ANSI AWS D17.1:2001, Specification for fusion welding aerospace applications.

[7] M. Corzo, O. Casals, J. Alcalá y A. Mateo. Rev. Metal. Madrid 41 (2005) 406-409.

[8] ASM Handbook Vol. 06: Welding, Brazing and Soldering, D.L. Olson, T.A. Siewert, S. Liu and G.R. Edwards (Eds.), ASM International, Ohio, EE. UU., 1993, pp. 1.289-1.319. 